\title{
Evaluation of multi drug resistance among extended spectrum \\ $\beta$-lactamase-producing Escherichia coli causing urinary tract infection in Erbil City \\ Received: 3/6/2014 \\ Accepted: 6/9/2014
}

\begin{tabular}{cc}
\hline Safaa Toma Aka * Sayran Hamad Haji * \\
\hline
\end{tabular}

Background and objective: Bacterial resistant to broad spectrum $\beta$-lactams, which is mediated by the extended spectrum beta lactamase enzyme, has emerged recently as increasing problem. Extended spectrum beta lactamase producing strains can also displaying multi-drug resistance. Thus, increased number of infections due to these strains is a public health issue associated with high morbidity, mortality, high health-care costs and prolonged hospitalization. Therefore, this study aimed to evaluate multi-drug resistance among extended spectrum beta lactamase producing E. coli causing urinary tract infections.

Methods: A total of 400 mid-stream urine specimens were collected from patients with urinary tract infection. Disk diffusion agar method on Muller-Hinton agar plates was carried out. Double Disc Synergy Test was used for detection of extended spectrum beta lactamase producer. All the isolates that were screened out for extended spectrum beta lactamase production were also subjected to confirmation by using the Phenotypic Confirmatory Combination Disc Diffusion Test.

Results: The urinary tract infection cases were mainly due to Gram negative bacteria $(87 \%)$. E. coli was isolated from $195(48 \%)$ specimens. Sixty isolates of E. coli $(31 \%)$ were found to be extended spectrum beta lactamase producers. The resistance to antibiotics tested was significantly higher $(P<0.001)$ among extended spectrum beta lactamase producing $\mathrm{E}$. coli isolates compared with non-extended spectrum beta lactamase producing isolates.

Conclusion: The prevalence of multi-drug resistance to the antibiotics among extended spectrum beta lactamase producing E. coli isolates was established. Imipenems are recommended for the treatment of serious infections caused by these organisms.

Keywords: E. coli, Eextended spectrum $\beta$-lactamase, Multi-drug resistance, DDST, PCDDT, Urinary tract infection.

\section{Introduction}

Extended spectrum beta lactamases (ESBL) enzymes were first described in Germany in 1983 from Klebsiella pneumonia. ESBL enzymes are usually plasmid mediated and gain broad resistance to cephalosporins: (cefotaxime, ceftazidime, ceftriaxone), and monobactams (aztreonam). ${ }^{1}$ A new class of ESBL, called CTX-M enzymes, has emerged during late 1990 and early 2000s, which was widely detected among Escherichia coli (E. coli) isolates. These ESBL-producing E. coli are able to resist penicillins, cephalosporins and are found mostly in urinary tract infections (UTI)., ESBL-producing strains can also display multi-drug resistance (MDR), including resistance to aminoglycosides and fluoroquinolones. Therefore, therapeutic options for these strains are limited. ${ }^{4-6}$ Continuous exposure of such bacterial strains to ß-lactams could induce mutation and production of new ß-lactamases, expand their activity even against the fourth generation cephalosporins. Thus, these new ß-lactamases are called extended spectrum ß-lactamases. ${ }^{7}$ ESBL-producing

* Department of Pharmacogonsy, College of Pharmacy, Hawler Medical University, Erbil, Iraq. 
organisms have been found most commonly in uropathogens like K. pneumoniae and E. coli. Other enterobacteria and non-fermenting Gram negative rods also produce ESBLs but to a lesser extent. ${ }^{8,9}$ In recent years, increasing ESBL- producers have been found in outpatient UTI, which aid the spreading isolates of MDR, ${ }^{10}$ which is defined as resistance to at least three classes of antimicrobial agents. ${ }^{11}$ Infections caused by MDR Gram negative bacteria have been associated with increased morbidity and mortality. ${ }^{12}$ It has been found that morbidity and mortality have increased also among UTI patients, especially those treated by inadequate antibiotics against ESBLs-producing E. coli. Thus, rapid detection of these isolates is essential for effective treatment. ${ }^{13}$ Various outbreaks of infection with ESBLs have been observed in many countries. These organisms are considered the main causative agents of nosocomial infections that lead to prolonged hospital stay. ${ }^{3}$ Studies reported detection of ESBL-producing Enterobacteriaceae in low rates of $3-8 \%$ in Sweden, Japan and Singapore compared to much higher prevalence rates documented in studies from Portugal (34\%), followed by Italy (37\%), USA (44\%), Latin American countries $(30-60 \%)$ and Turkey $(58 \%)$. While detection rates ranged from 8.5-38.5\% have been found in the Kingdom of Saudi Arabia and (31.7\%) in Kuwait; the highest level of $41 \%$ is from the United Arab Emirates. ${ }^{14}$ This study aimed to evaluate the prevalence of MDR among ESBL-producing and non-ESBL producing $\mathrm{E}$. coli isolated from urine cultures in patients attending Rezgari Teaching Hospital in Erbil City.

\section{Methods}

\section{Specimen collection and bacterial} isolates

This study was conducted at Rizgary Teaching Hospital in Erbil City, Iraqi Kurdistan Region, during the period from October 2012 to August 2013. A total of 400 mid-stream urine specimens were collected from patients suffering from UTI. Urine culture was done using standard wire loop $0.001 \mathrm{ml}$. It was streaked on $5 \%$ sheep blood agar and MacConkey agar plates. The inoculated plates were incubated aerobically at $37^{\circ} \mathrm{C}$ for $24-48$ hrs. $^{15}$ Identification was done on the basis of colony morphology, Indole test, Simmon's citrate agar and standard biochemical tests. Identification of isolates was confirmed using $\mathrm{API}-20 \mathrm{E}$ test (bioMerieux). Only significant bacterial growth $\left(>10^{5} \mathrm{cfu} / \mathrm{ml}\right)$ were included in the study. $^{5}$

Antimicrobial Susceptibility Test (AST)

Disk diffusion agar method on MullerHinton agar (MHA) plate was achieved. The results were interpreted according to Clinical Laboratory and Standards Institute (CLSI) guidelines. ${ }^{16}$ The following antimicrobials from (Rashmi Diagnostic Ltd., India) were tested: ampicillin $(10 \mu \mathrm{g})$, cefixime $(30 \mu \mathrm{g})$, gentamycin $(10 \mu \mathrm{g})$, cotrimoxazole $(25 \mu \mathrm{g})$, ciprofloxacin $(10 \mu \mathrm{g})$, amoxicillin/clavulanic acid (amoxiclav) $(20 / 10 \mu \mathrm{g})$, aztreonam $(30 \mu \mathrm{g})$, ceftriaxone $(30 \mu \mathrm{g})$, ceftazidime $(30 \mu \mathrm{g})$, cefotaxim $(30 \mu \mathrm{g}), \quad$ nitrofurantion $(10 \mu \mathrm{g})$, piperacillin $(25 \mu \mathrm{g})$, and imipenem $(10 \mu \mathrm{g})$. All E. coli isolates that showed resistant to at least three different classes of antimicrobial agents were determined as MDR. ${ }^{5}$

\section{Detection of ESBL isolate}

National Committee of Clinical Laboratory Slandered (NCCLS) screening test

Isolates showing an inhibition zone size of $\leq 22 \mathrm{~mm}$ to ceftazidime $(30 \mu \mathrm{g}), \leq 25 \mathrm{~mm}$ to cefriaxone $(30 \mu \mathrm{g})$ and $\leq 27 \mathrm{~mm}$ with cefotaxime $(30 \mu \mathrm{g})$ were identified as potential ESBL-producing isolates and were short-listed for confirmation of ESBL- production.

\section{Double Disk Synergy Test (DDST)}

All isolates showing resistance to one or more of the third generation cephalosporins were tested for ESBLproduction, using a double disk synergy test (DDST) as a standard disk-diffusion assay on MHA plates. The amoxicillin/ clavulanic acid $(20 / 10 \mu \mathrm{g})$ was placed in the 
center of the plate, and the following disks of $\beta$-lactam antibiotics were placed $15 \mathrm{~mm}$ apart (edge to edge) from the center in order to observe the synergistic effect: cefotaxime $(30 \mu \mathrm{g})$, ceftazidime $(30 \mu \mathrm{g})$, ceftriaxone $(30 \mu \mathrm{g})$ and aztreonam $(30 \mu \mathrm{g})$. Following incubation, a clear extension of the edge of the inhibition zone of cephalosporin toward the augmenting disk was interpreted as positive for ESBL production. $^{17,18}$

\section{Phenotypic Confirmatory Combination Disk Diffusion Test (PCDDT)}

All the isolates that were screened out for ESBL-production were also subjected to confirmation by using the PCDDT. ${ }^{1,17}$ The disks of cefotaxime $(30 \mu \mathrm{g})$, cefotaxime/clavulanic acid (20/10 $\mu$ g), ceftazidime $(30 \mu \mathrm{g})$ and ceftazidime / clavulanic acid $(20 / 10 \mu \mathrm{g})$ were placed on the MHA plates. Following incubation, $a \geq 5 \mathrm{~mm}$ increase in diameter of the inhibition zone of the cephalosporin/ clavulanic disk when compared to the cephalosporin disc alone were interpreted as phenotypic evidence of ESBLproduction. $^{19}$

\section{Statistical analysis}

The mean $\pm S D$ of antibiotic resistance were estimated and the paired sample t-test was used to compare these means. $\mathrm{P}$-value $<0.05$ was considered statistically

\section{Results}

\section{Clinical isolates}

A total of 400 urine specimens were included in the study. Majority $(87 \%)$ of UTI were due to Gram negative bacteria, while remaining $13 \%$ were due to Gram positive bacteria. E. coli was present in $195(48 \%)$ of specimens, representing the most common isolates.

\section{Detection of ESBLs}

Of 195 clinical isolates of E. coli, only $60(31 \%)$ showed to be ESBL-producers, while $135(69 \%)$ were non ESBLproducers. DDST could detect only 55 ESBL-producers, whereas a total of 60 ESBL were detected by PCDDT. The sensitivity and specificity of DDST were $91.66 \%$ and $96.26 \%$, respectively as shown in Table 1.

Table 1: Sensitivity and specificity of DDST in comparison with PCDDT for detection of ESBL-producing E. coli.

\begin{tabular}{|c|c|c|c|c|c|}
\hline \multirow{2}{*}{$\begin{array}{l}\text { Detection of } \\
\text { ESBL-Producing-E.coli } \\
(n=195)\end{array}$} & \multicolumn{2}{|c|}{ PCDDT positive } & \multicolumn{2}{|c|}{ PCDDT negative } & \multirow{2}{*}{ Total } \\
\hline & $n=60$ & $(\%)$ & $n=135$ & $(\%)$ & \\
\hline DDST positive & 55 & $(91.66 \%)$ & 5 & $(3.73 \%)$ & 60 \\
\hline DDST negative & 5 & $(8.33 \%)$ & 130 & $(96.26 \%)$ & 135 \\
\hline Total & 60 & $(100 \%)$ & 135 & $(100 \%)$ & 195 \\
\hline
\end{tabular}

Sensitivity: $91.66 \% \&$ Specificity: $96.26 \%$ 
Antibiotic susceptibility

All ESBL-producing E. coli were 100\% resistant to ampicillin, while $(98 \%)$ and $(96 \%)$ showed resistant to ceftazidime and cefotaxime, respectively. All isolates were sensitive $(100 \%)$ to imipenem. Non ESBL-producing E. coli exhibited high resistance to ampicillin (85\%), followed by amoxiclave (81\%), and piperacillin $(50 \%)$, while all were sensitive to imipenem (Figure 1). ESBL-producing E. coli could display significant resistance $(P<0.001)$ to 13 types of antibiotics compared with Non-ESBL-producers as shown in Table 2.

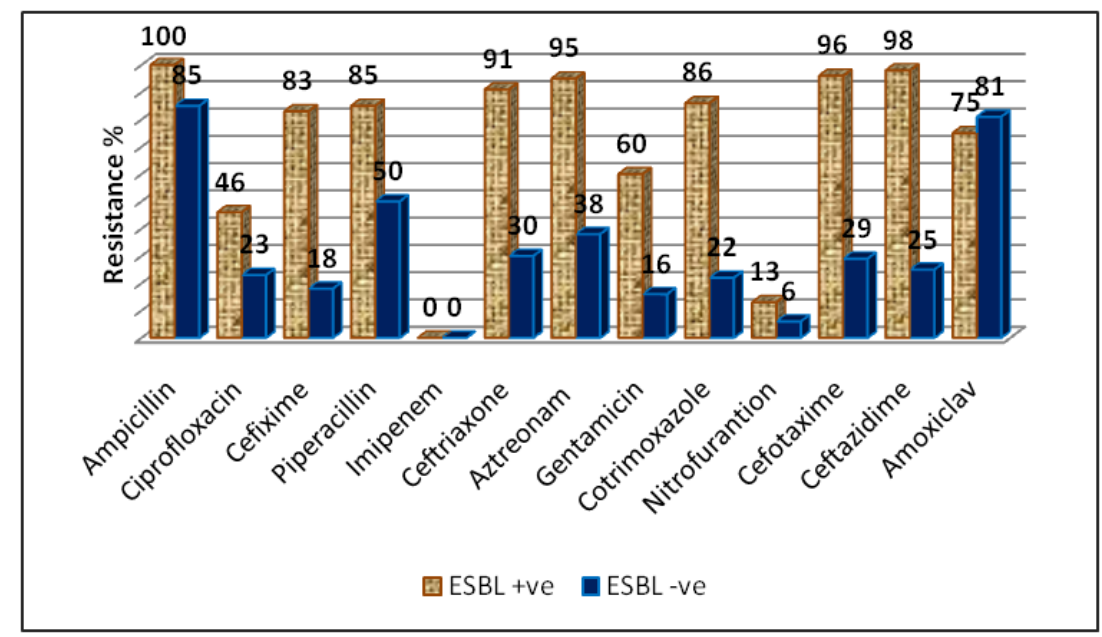

Figure 1: The percentage of resistance among ESBL-producers and non ESBL-producers E. coli isolates.

Table 2: Number and percentage of antimicrobial resistant of both ESBL-producers and non ESBL-producing E. coli isolates.

\begin{tabular}{lllll}
\hline Antibiotics & $\begin{array}{l}\text { ESBL-Producer } \\
\mathbf{n = 6 0}\end{array}$ & Mean \pm SD & $\begin{array}{l}\text { Non-ESBL-Producer } \\
\mathbf{n = 1 3 5}\end{array}$ \\
\hline Ampicillin & $60(100 \%)$ & $99.73 \pm 0.64^{*}$ & $115(85 \%)$ & $85.07 \pm 0.80^{*}$ \\
Ciprofloxacin & $28(46 \%)$ & $45.38 \pm 0.63^{*}$ & $32(23 \%)$ & $23.30 \pm 0.62^{*}$ \\
Cefixime & $50(83 \%)$ & $83.48 \pm 1.02^{*}$ & $25(18 \%)$ & $18.115 \pm 0.33^{*}$ \\
Piperacillin & $51(85 \%)$ & $85.22 \pm 1.02^{*}$ & $68(50 \%)$ & $50.27 \pm 0.067^{*}$ \\
Imipenem & 0 & 0 & 0 & 0 \\
Ceftriaxone & $55(91 \%)$ & $91.48 \pm 0.89^{*}$ & $41(30 \%)$ & $31.0 \pm 1.0^{*}$ \\
Aztreonam & $57(95 \%)$ & $95.26 \pm 1.08^{*}$ & $52(38 \%)$ & $38.16 \pm 0.9^{*}$ \\
Gentamicin & $30(60 \%)$ & $60.34 \pm 1.41^{*}$ & $21(16 \%)$ & $16.32 \pm 0.58^{*}$ \\
Cotrimoxazole & $52(86 \%)$ & $86.4 \pm 1.32^{*}$ & $30(22 \%)$ & $22.21 \pm 1.2^{*}$ \\
Nitrofurantion & $8(13 \%)$ & $13.13 \pm 1.15^{*}$ & $9(6 \%)$ & $6.17 \pm 1.04^{*}$ \\
Cefotaxime & $58(96 \%)$ & $96.18 \pm 1.2^{* *}$ & $40(29 \%)$ & $29.18 \pm 1.07^{* *}$ \\
Ceftazidime & $59(98 \%)$ & $98.19 \pm 1.18^{*}$ & $35(25 \%)$ & $25.44 \pm 1.45^{*}$ \\
Amoxiclav & $45(75 \%)$ & $75.33 \pm 1.18^{* *}$ & $110(81 \%)$ & $81.10 \pm 0.97^{* *}$ \\
\hline
\end{tabular}

All data were expressed as mean $\pm S D$; the mean difference is significant at $\left(^{* *}\right) p<0.01$ and $\left({ }^{*}\right)$ at $p<0.001$ 
Multi-drug resistance was found to be greater among ESBL-producing isolates, showing resistance against 12 types of antibiotics as summarized in Figure 2.

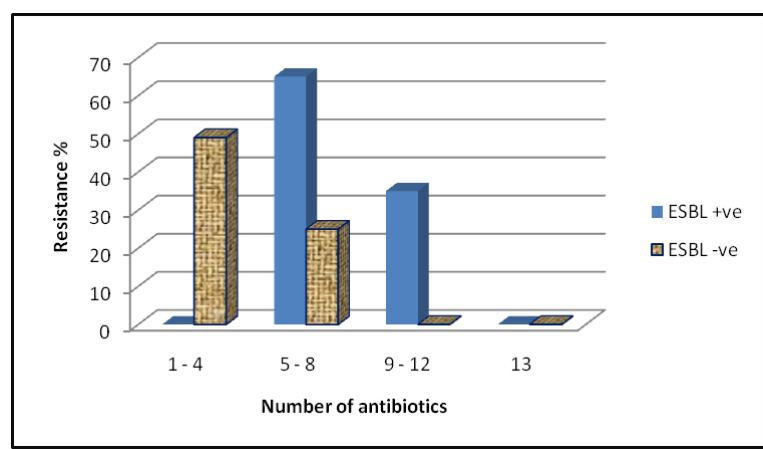

Figure 2: The percentage of MDR pattern (\%) among clinical isolates of $E$. coli with ESBL and non ESBL-producers.

\section{Discussion}

This study showed the bacterial isolates involved in UTIs were mainly Gram negative bacteria (87\%); the significant growth for $E$. coli was present in $48 \%$ of urine specimen. It is believed that uropathogenic E. coli reside in the colon, and are later introduced through the urethra to the bladder. ${ }^{20}$ This work was designed to examine all $\mathrm{E}$. coli strains isolated from urine specimens to study the prevalence of ESBL-producing isolates, since these pathogens are now recognized worldwide as most important causative agents of nosocomial and communityacquired infections. ${ }^{14}$ ESBLs-producing E. coli represented $31 \%$. Similar rates were reported from Kufa City, the southern Iraq (38\%) and Iran (35\%). ${ }^{21}$ A study from Turkey also reported a rate of $25 \%$ of infection with ESBL-producing Gram negative bacteria. $^{22}$ The antimicrobial resistance displayed various results. All ESBL-producing E. coli were significantly resistant to $3^{\text {rd }}$ and $4^{\text {th }}$ generations cephalosporins: ceftazidime (98\%), cefotaxime $(96 \%)$, ceftriaxone $(91 \%)$, and cefixime $(83 \%)$. These results are consistent with the related studies in the same field. ${ }^{7,13,23}$ Furthermore, ESBL-producing E. coli was highly resistant to amoxiclav (75\%). These finding might be due to long term uses as empirical therapy to UTIs. Multi-drug resistant isolates showed to be greater among ESBL-producing $E$. coli than non ESBL-producers. Similar findings were reported in a number of recent studies. ${ }^{7,13}$, 24 In fact, ESBL-producing organisms of the family Enterobacteriaceae were primarily considered MDR initiated in hospitals. In recent years, an increase of such ESBL-producers has been observed in outpatient settings, especially related to UTIs, reducing the treatment options to a limited number of antibiotics. ${ }^{10}$ Treatment of the infections caused by ESBLproducing organisms is not easy. This is because of the resistance to the extended spectrum cephalosporins themselves, and also associated with resistance to other antimicrobial agents coded by plasmids. ${ }^{25}$ Therefore, an increase in the number of infections due to MDR organisms is a public health issue associated with high morbidity, mortality, high health-care cost and prolonged hospitalization. ${ }^{19}$ In view of the fact that ESBL-producing organisms are MDR, therapeutic selections for these infections are limited. In recent years, imipenem has been recommended for treatment of infections caused by ESBL-producing organisms. Because of increasing incidence and pathogenic significance of ESBL-producing $E$. coli in community and nosocomial infections, gathering additional data on clinical risk factors for these isolates are recommended. ${ }^{26}$

\section{Conclusion}

Prevalence of MDR to the antibiotics among $\mathrm{ESBL}$-producing $\mathrm{E}$. coli isolates was established. The study suggests regular screening and surveillance for ESBL-producing E. coli. Imipenems are recommended for the treatment of serious infections caused by these organisms.

\section{Conflicts of interest}

The authors report no conflicts of interest. 


\section{References}

1. Altayar MA, Thokar MA, Mohammad MA. Extended spectrum B-lactamase-producing Escherichia coli in clinical isolates in Benghazi, Libia: Phenotypic detection and antimicrobial susceptibility pattern. Medical Journal of Islamic World Academy of Sciences 2012; 20(2):49-56.

2. Livermore $\mathrm{DM}$, Canton $\mathrm{R}$, Gniadkowski $\mathrm{M}$, Nordmann P, Rossolini GM, Arlet G, et al. CTX-M: changing the face of ESBLs in Europe. J Antimicrob Chemother 2007; 59(2):165-74.

3. Hassan MI, Alkharsah KR, Alzahrani AJ, Obeid $\mathrm{OE}$, Khamis $\mathrm{AH}$, Diab A. Detection of extended spectrum beta-lactamases-producing isolates and effect of AmpC overlapping. J Infect Dev Ctries 2013; 7(8):618-29.

4. Tukaram V, Nilekar S, Shivraj NK. ESBLs-A Challenge to the Clinicians. Indian Medical Gazette 2012; 22(2):87-91.

5. kreem Al-salamy A. Detection of extended spectrum-beta lactamase enzymes producing E. coli that isolated from urine. Kufa Journal For Veterinary Medical Sciences 2012; 3(1):55-66.

6. Gundogan N, Avci E. Prevalence and antibiotic resistance of extended-spectrum beta-lactamase (ESBL) producing Escherichia coli and Klebsiella species isolated from foods of animal origin in Turkey. Afr J Microbiol Resch 2013; 7(31): 4059-64.

7. Dalela G. Prevalence of Extended Spectrum Beta Lactamase (ESBL) Producers among Gram Negative Bacilli from Various Clinical Isolates in a Tertiary Care Hospital at Jhalawar, Rajasthan, India. J Clin Diagn Res 2012; 6:182-7.

8. Egbebi A, Famurewa O. Prevalence of extended spectrum beta-lactamase (ESBL) production among Klebsiella isolates in some parts of South West Nigeria. Microbiol Biotech Res 2011; 1(2): 64-8.

9. Ejaz H. Detection of extended-spectrum $\beta$ lactamases in Klebsiella pneumoniae: Comparison of phenotypic characterization methods. Pak J Med Sci 2013; 29(3):768-72.

10. Auer S, Wojna A, Hell M. Oral treatment options for ambulatory patients with urinary tract infections caused by extended-spectrum- $\beta$ lactamase-producing Escherichia coli. Antimicrob Agents Chemother 2010; 54(9):4006-8.

11. Paterson DL. The epidemiological profile of infections with multidrug-resistant Pseudomonas aeruginosa and Acinetobacter species. Clin Infect Dis 2006; 43(Supplement 2):S43-8.

12. Amutha $R$, Murugan $T$, Devi MR. Studies on multidrug resistant Pseudomonas aeruginosa from pediatric population with special reference to extended spectrum beta lactamase. Indian Journal of Science and Technology 2009; 2(11):11-3.

13. Batarseh A, Soneah S, Mardeni R, Elmadni K, Noor M, Abu Ashour N. Antibiotic resistance pattern of multidrug resistant and extendedspectrum beta-lactamaseproducing-Escherichia coli urinary isolates at Queen Rania Al-Abdullah Hospital for children. Jordan.Z.U.M.J 2013; 19(5).

14. Khanfar HS, Bindayna KM, Senok AC, Botta GA. Extended spectrum beta-lactamases (ESBL) in Escherichia coli and Klebsiella pneumoniae: trends in the hospital and community settings. J Infect Dev Ctries 2009; 3(4):295-8.

15. Leong A. Principles and Practice of Medical Laboratory Sciences. New York, Churchill Livingston; 1997.

16. Dowzicky MJ, Park $\mathrm{CH}$. Update on antimicrobial susceptibility rates among gram-negative and gram-positive organisms in the United States: results from the Tigecycline Evaluation and Surveillance Trial (TEST) 2005 to 2007. Clin Ther 2008; 30(11):2040-50.

17. Giriyapur RS, Nandihal NW, Krishna B, Patil AB, Chandrasekhar M. Comparison of disc diffusion methods for the detection of extended-spectrum beta lactamase-producing Enterobacteriaceae. J Lab Physicians 2011; 3(1):33-6.

18. Drieux L, Brossier F, Duquesnoy O, Aubry A, Robert J, Sougakoff $W$, et al. Increase in hospital-acquired bloodstream infections caused by extended spectrum $\beta$-lactamase-producing Escherichia coli in a large French teaching hospital. Eur J Clin Microbiol Infect Dis 2009; 28 (5):491-8.

19. Chaudhary N, Murthy S. Prevalence of Multidrug Resistance in Uropathogenic Klebsiella Species With Reference to Extended Spectrum $\beta$-Lactamases Production. Research Journal of Pharmaceutical, Biological and Chemical Sciences 2013; 4(3):728-35.

20. Jantunen M, Saxen H, Lukinmaa S, Ala-Hauhala M, Siitonen A. Genomic identity of pyelonephratogenic Escherichia coli isolated from blood, urine and faeces of children with urosepsis. J Med Microbiol 2001; 50:650-2.

21. Behrouzi A, Rahbar M, Vandy and Yousefi J. Frequency of extended spectrum beta-lactamase (ESBLs) producing Escherichia coli and Klebseilla pneumonia isolated from urine in an Iranian 1000-bed tertiary care hospital. African Journal of Agriculture research 2010; 9:881-4.

22. Gedik H. Risk factors for drug resistant bacterial infections: Hospital based study. Afr J Microbiol Res 2012; 6(6):1190-6.

23. Ejaz H, Zafa A, Mahmood S, Javed MM. Urinary tract infections caused by extended spectrum $\beta$-lactamase (ESBL) producing Escherichia coli and Klebsiella pneumoniae. African Journal of Biotechnology 2013; 10(73):16661-6.

24. Nimri L, Azaizeh B. First Report of MultidrugResistant ESBLProducing Urinary Escherichia coli in Jordan. British Microbiology Research Journal 2012; 2(2):71-81.

25. Tumbarello M, Citton R, Spanu T, Sanguinetti M, Romano L, Fadda G, et al. ESBL-producing 
Evaluation of multi drug resistance among .....

Zanco J. Med. Sci., Vol. 19, No. (2), 2015

http://dx.doi.org/10.15218/zjms.2015.0023

multidrug-resistant Providencia stuartii infections in a university hospital. J Antimicrob Chemother 2004; 53(2):277-82.

26. Kang C-I, Wi YM, Lee MY, Ko KS, Chung DR, Peck KR, et al. Epidemiology and risk factors of community onset infections caused by extendedspectrum $\beta$-lactamase-producing Escherichia coli strains. J Clin Microbiol 2012; 50(2):312-7. 